\title{
Single-electron transistors with wide operating temperature range
}

C. Dubuc, J. Beauvais, and D. Drouin

Citation: Appl. Phys. Lett. 90, 113104 (2007); doi: 10.1063/1.2713171

View online: http://dx.doi.org/10.1063/1.2713171

View Table of Contents: http://aip.scitation.org/toc/apl/90/11

Published by the American Institute of Physics

\section{Articles you may be interested in}

Room-temperature Al single-electron transistor made by electron-beam lithography Applied Physics Letters 76, 2256 (2000); 10.1063/1.126313

\section{AlP $\mid$ Applied Physics AlP I Letters}

Save your money for your research.

It's now FREE to publish with us -

no page, color or publication charges apply.

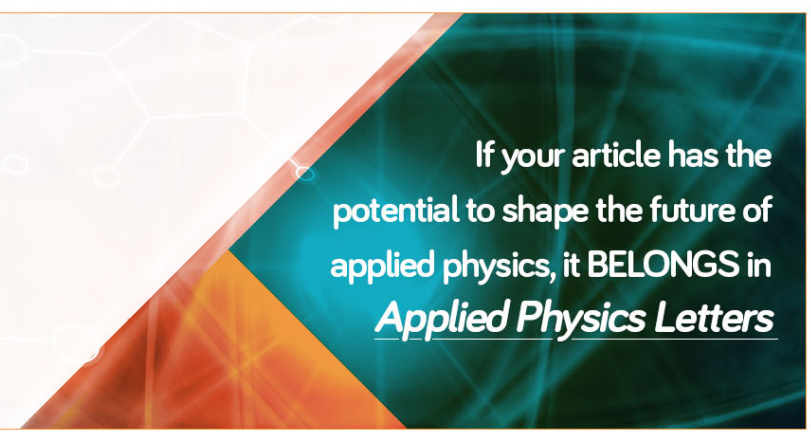




\title{
Single-electron transistors with wide operating temperature range
}

\author{
C. Dubuc, ${ }^{\text {a) }}$ J. Beauvais, and D. Drouin \\ Department of Electrical Engineering, University of Sherbrooke, 2500 Boulevard de l'Université, \\ Sherbrooke, Quebec J1K 2R1, Canada
}

(Received 24 October 2006; accepted 6 February 2007; published online 12 March 2007)

\begin{abstract}
Single-electron transistors are fabricated with a planar self-aligned process using chemical mechanical polishing. The method is demonstrated with $\mathrm{Ti} / \mathrm{TiO}_{x}$ junctions and resistless lithography. The device characterization showed Coulomb blockade up to $433 \mathrm{~K}$. High temperature data allowed one to calculate the impact of the process variations on the charging energy and thus on a realistic operating temperature. It is found that single electron devices can have an operating temperature range similar to conventional silicon transistors, opening the door to hybrid designs. These approaches are promising because advanced functionality is created by an optimal combination of both technology strengths. (C) 2007 American Institute of Physics.
\end{abstract}

[DOI: $10.1063 / 1.2713171]$

Single electron transistors (SETs) have been around for more than two decades and their potential as extremely high density devices has already been established as well as their unique characteristics for niche markets. ${ }^{1,2}$ The nanometer scale dimensions of these transistors have represented an important fabrication challenge and still remain a major hurdle to their widespread utilization. Room temperature demonstration was an important milestone in SETs history, but it is not clear whether it is a sufficient condition for their integration into advanced applications. Like any other electronic device, SETs need to operate properly over a wide range of conditions. More specifically, SETs require an acceptable operating temperature range that will guarantee a sufficient tolerance to normal fabrication process variations and operational heat dissipation. A wider utilization of SETs also supposes a fabrication process that can be transferred into mass production methods.

Many SET processes compatible with the manufacturing environment were presented over recent years. ${ }^{3,4}$ While some exhibited relatively high charging energy, few were characterized above room temperature, providing very little data on SETs actual tolerance for operation in real environment. Hybrid approaches, ${ }^{5,6}$ where SETs and conventional silicon field effect transistors (Si-FETs) are used in combination on the same substrate, are attractive because these circuits can take advantage of both device's strengths to create advanced functionality. Again, this approach requires SETs with an operational temperature range similar to Si-FETs which has not been specifically demonstrated in previous work.

Here we present a SET concept and its related fabrication that was used to produce devices with a wide temperature range. It is based on a damascene approach using electron beam lithography (EBL) and chemical mechanical polishing (CMP), both technologies which are already in use in the manufacturing environment. The fabricated prototypes have sufficient built-in tolerance to sustain fabrication process variations and still function within a temperature range similar to other conventional devices such as Si-FETs. A very unique tunnel junction creation method is used to control exactly the dielectric thickness and the junction locations.

\footnotetext{
a)Electronic mail: dubuc@emt.inrs.ca
}

The demonstration is made with metallic SETs using the $\mathrm{Ti} / \mathrm{TiO}_{x}$ system at the junctions.

The fabrication process begins with high resolution patterning of a silicon wafer coated with a thermally grown $\mathrm{SiO}_{2}$ layer. We have selected an EBL process that does not use organic resist for patterning. Inorganic layers such as $\mathrm{SiO}_{2}$ are good candidates for sub-10 nm imaging ${ }^{7}$ and simplify the CMP process. The electron beam directly irradiates the $\mathrm{SiO}_{2}$ layer, modifying its density ${ }^{8}$ and, as a consequence, its etch rate in a dilute acid solution. The irradiated areas etch faster than the background, leading to trench formation. The trenches are not designed to reach the silicon substrate and the underlying oxide ensures electrical isolation from the substrate. When this isolation layer is reduced to a few tens of nanometers in thickness, it can also be used as a back-gate dielectric layer. The shape of the device after this step is a narrow trench in the oxide less than $15 \mathrm{~nm}$ wide by $25 \mathrm{~nm}$ deep, as shown in Fig. 1(a). The depth of the trench is entirely controlled by the lithography exposure dose and can be precisely adjusted according to design needs.

Figure 1(b) shows how the SET island is created by patterning a $\mathrm{Ti}$ line perpendicular to the oxide trench. The line is oxidized in pure oxygen to grow 2 to $12 \mathrm{~nm}$ of $\mathrm{TiO}_{x}$ depending on oxidation time and temperature. $\mathrm{This}^{\mathrm{TiO}_{x}}$ on the Ti line sidewalls constitutes the tunnel junction dielectric layer. The width of the Ti line will determine the length of the SET island and contributes to its self-capacitance. This line can be patterned with reduced resolution requirements without compromising the total SET capacitance $C_{T}$, which must meet the subattofarad target required for wide range temperature operation. This relaxation of the resolution requirement is due to the fact that the tunnel junction capacitances of this process are extremely small as a result of the self-aligned fabrication technique. This represents a key element of this SET concept. In this work, EBL combined with a lift-off technique was used for patterning the Ti lines, with a resolution ranging from 50 to $75 \mathrm{~nm}$.

In the last process step, a Ti layer called the blanket layer is deposited over the entire device area, as shown in Fig. 1(c). The substrate is fixed to a polishing jig and put face down on a turning polishing pad for chemical mechanical polishing. This planarization process will eliminate all material located above the surface of the thermally grown $\mathrm{SiO}_{2}$ 
(a)

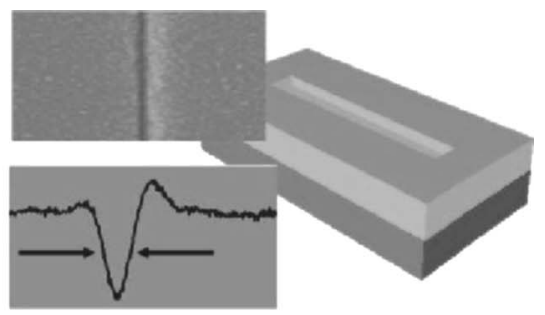

(b)

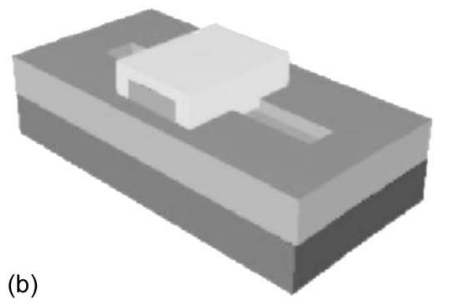

(c)
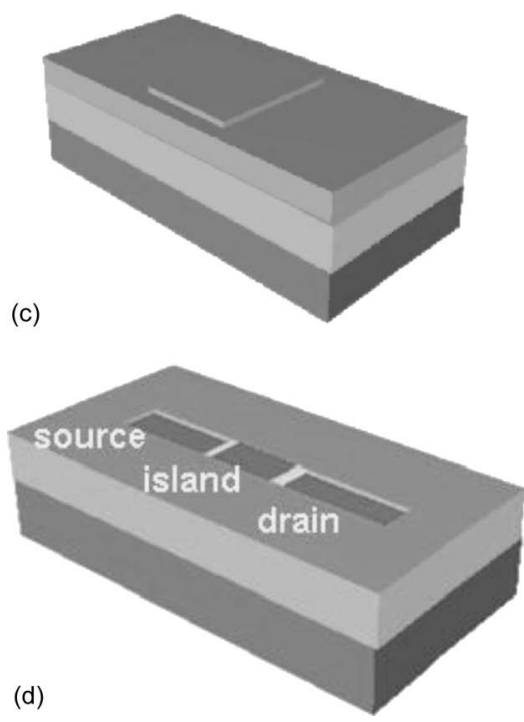

FIG. 1. Wide temperature SETs process overview. (a) View of the trench in the oxide layer and its top down SEM picture including the intensity profile showing where the linewidth is measured. Below the oxide layer is the silicon substrate used as a back gate. (b) The island formation. The open end drawing of the perpendicular $\mathrm{Ti}$ line shows the $\mathrm{TiO}_{x}$ layer grown on the future island. (c) The titanium blanket layer is deposited. (d) End result after CMP.

layer. The top of the island is thus removed but its $\mathrm{TiO}_{x}$ sidewalls continue to isolate it from the rest of the thin line, which is now embedded in the $\mathrm{SiO}_{2}$. The end result is presented in Fig. 1(d). The CMP process is stopped when test structures placed next to the SETs begin to disappear. This is because they intentionally received a lower exposure dose and are thus a few nanometers shallower that the SETs trench. We are investigating the use of stopping layers to improve the polishing accuracy, but with the current method, this optical end point strategy was quite easy to detect and control. The CMP process leads to the simultaneous formation of the source, drain, and island of the SETs with a thickness of only $2-3 \mathrm{~nm}$. Depending on the specific design of the devices, larger source and drain electrodes can then be deposited to facilitate the electrical characterization of the SETs.

The source-drain current $I_{\mathrm{DS}}$ measured at room temperature is presented in Fig. 2(a) for two different back-gate bias values turning the device on and off. The inset of Fig. 2(a) shows the SET characteristics under a wider $V_{\mathrm{DS}}$ range. The behavior of the $I_{\mathrm{DS}}-V_{\mathrm{DS}}$ curves is strongly nonlinear for both
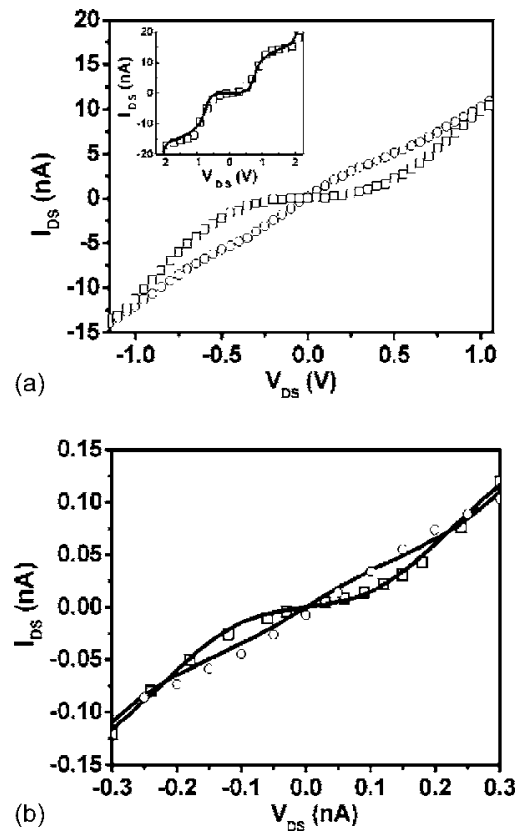

FIG. 2. Electrical characterization at room temperature. (a) Asymmetrical SET data with $V_{\mathrm{GS}}$ at $0 \mathrm{~V}(\square)$ and at $0.3 \mathrm{~V}(\mathrm{O})$. The inset shows a wider $V_{\mathrm{DS}}$ range $(\square)$. The line represents the model calculations with $C_{\mathrm{S}}=C_{\mathrm{D}}$ $=0.06 \mathrm{aF}, R_{\mathrm{S}}=1.7 \times 10^{7} \Omega, R_{\mathrm{D}}=3.5 \times 10^{6} \Omega$, and $C_{\mathrm{GS}}=0.23 \mathrm{aF}$. (b) Symmetrical SET data with tunnel junction dielectric thickness of $12 \mathrm{~nm}$ for $V_{\mathrm{GS}}$ at $0 \mathrm{~V}(\square)$ and at $0.3 \mathrm{~V}(\bigcirc)$. The line represents the model calculation with $C_{\mathrm{S}}=C_{\mathrm{D}}=0.05 \mathrm{aF}, R_{\mathrm{S}}=6.3 \times 10^{8} \Omega, R_{\mathrm{D}}=8.9 \times 10^{8} \Omega$, and $C_{\mathrm{GS}}=0.3 \mathrm{aF}$.

symmetrical and asymmetrical transistors with relatively high current drive in the nanoampere range. The asymmetrical case is a design with SET neighboring features that create localized uneven polishing, resulting in rounding of one of the junctions and thus in conductance differences. Other $I_{\mathrm{DS}}-V_{\mathrm{DS}}$ curves were obtained on a symmetrical layout and one example is presented in Fig. 2(b). We also scanned the back-gate bias and observed a transistor effect. The drain current is modulated by the back-gate voltage and showed regular oscillations, as presented in Fig. 3. We have observed a back-gate leakage through the backside oxide on the samples with sub-100 nm thickness. The leakage is a back gate to source current of about $100 \mathrm{pA} / V_{\mathrm{GS}}$ and occurred after several temperature cycling tests. Metallic SETs are known to be surprisingly well described within a certain size range by a set of basic rules called the orthodox theory. ${ }^{9,10}$ To model the devices at room temperature, we have used Monte Carlo simulations within this framework with capacitance

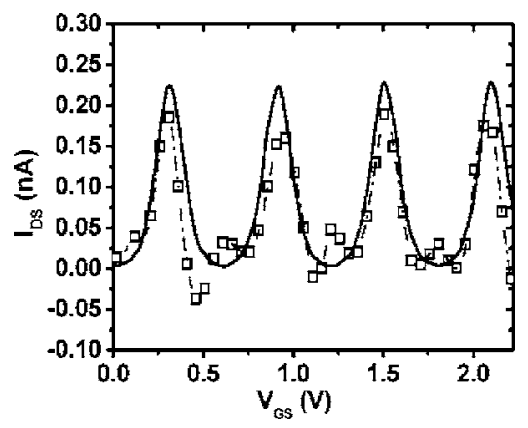

FIG. 3. SET drain current behavior as a function of the back-gate bias at low drain voltage $(25 \mathrm{mV})$. The line is the asymmetrical SET model and the squares $(\square)$ are the data. A dotted line is added as a guide to the eye. A gate to source leakage to of $100 \mathrm{pA} / \mathrm{V}_{\mathrm{GS}}$ was removed from the data to represent it on the same scale as the model. 


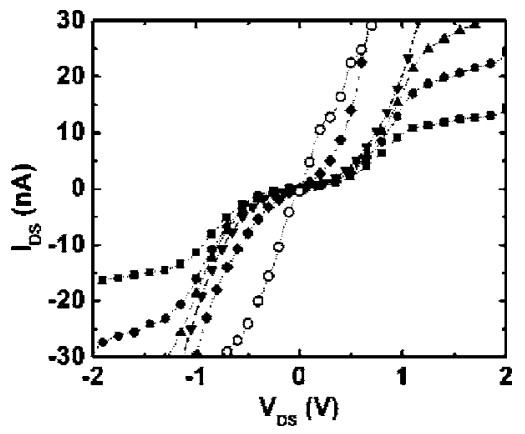

FIG. 4. SET curves as a function of temperature for the design with the charging energy of $457 \mathrm{meV}$. Temperatures are $296 \mathrm{~K}(\boldsymbol{\nabla}), 316 \mathrm{~K}(\bullet)$, $336 \mathrm{~K}(\boldsymbol{\Delta}), 386 \mathrm{~K}(\boldsymbol{\nabla})$, and $433 \mathrm{~K}(\bullet)$. The back gate is grounded except for the open circles where $V_{\mathrm{GS}}$ is $0.3 \mathrm{~V}$ at $433 \mathrm{~K}$.

and resistance values close to what is expected from the geometry of the devices. The dimensions were extracted from measurements using a scanning electron microscope (SEM) and the oxide thickness was verified by ellipsometry. In the case of the asymmetrical transistor of Fig. 2(a), the junction dimensions were $10 \mathrm{~nm}$ wide by $2 \mathrm{~nm}$ thick with an $8 \mathrm{~nm}$ layer of $\mathrm{TiO}_{x}$ grown at low temperature. The dielectric constant of 3.5 of the titanium oxide was extracted from junction current curves as a function of electric field and temperature. This value is typical of an amorphous $\mathrm{TiO}_{x}$ thin film. ${ }^{11,12}$ With a parallel plate model we evaluated the gate capacitance at $2.3 \times 10^{-19} \mathrm{~F}$ and the source and drain capacitances at $7.7 \times 10^{-20} \mathrm{~F}$. Considering the relative measurement error of the SEM at this small size scale, these values are in good agreement with the model values which are $2.3 \times 10^{-19} \mathrm{~F}$ for the gate capacitance and $6.0 \times 10^{-20} \mathrm{~F}$ for the source and drain capacitances. These results confirmed that this selfaligned junction fabrication process is capable of subattofarad resolution. We have explored many scenarios and combinations of possible unexpected multiple junctions and conductance but none of them could explain our data other than Coulomb blockade arising from standard SET formation. With this model, we have estimated our charging energy at $457 \mathrm{meV}$.

The $I_{\mathrm{DS}}-V_{\mathrm{DS}}$ curve was then characterized as a function of temperature and is presented in Fig. 4. Measurements on the design with the highest charging energy were made up to the limit of the experimental setup at $433 \mathrm{~K}$ and the nonlinearity attributed earlier to Coulomb blockade was still being observed. This is because the charging energy $E_{C}$ of $457 \mathrm{meV}$ is still more than 12 times the value of thermal fluctuations at $433 \mathrm{~K}$. Taking this same factor of 12 as a criterion for an initial approximation, one finds that even if some fabrication steps deviate from nominal values and reduce $E_{C}$ by $25 \%$ to $343 \mathrm{meV}$, Coulomb blockade could still be observable up to around $330 \mathrm{~K}$. This temperature reaches the upper specification limit of normal operation of many $\mathrm{Si}$-FETs processes. It is then clear in this case that the SET operating temperature alone is not the limiting factor in hybrid design fabrication. This conclusion is important for circuit designers because it sets a value above room temperature under which Coulomb blockade can be guaranteed.

The first three designs fabricated with this approach were all functional devices. A larger number of SETs based on this approach will need to be fabricated in order to evaluate the complete yield and repeatability of the process. Nevertheless, as an initial step, we have demonstrated the proof of concept of this method. Getting high temperature data gave us a valuable insight on how SETs evolve above room temperature and what kind of process tolerances will be required to establish hybrid circuit fabrication. We also believe that this device platform might have some use to study and validate concepts of single charge tunneling theory at very high temperature.

The authors are grateful for grants from the Natural Sciences and Engineering Research Council of Canada. The authors also thank NanoQuébec and the Regroupement Québécois sur les Matériaux de Pointe for additional support for the nanofabrication infrastructure.

${ }^{1}$ K. Likharev, Proc. IEEE 87, 606 (1999).

${ }^{2}$ Y. Takahashi, Y. Ono, A. Fujiwara, and H. Inokawa, J. Phys.: Condens. Matter 14, R995 (2002).

${ }^{3}$ K. Miyaji, M. Saitoh, and T. Hiramoto, Appl. Phys. Lett. 88, 143505 (2006).

${ }^{4}$ Y. Ono, Y. Takahashi, K. Yamazaki, M. Nagase, H. Namatsu, K. Kurihara, and K. Murase, IEEE Trans. Electron Devices 47, 147 (2000).

${ }^{5}$ A. Toriumi, K. Uchida, R. Ohba, and J. Koga, Physica B 272, 522 (1999).

${ }^{6}$ K. S. Park, S.-J. Kim, I.-B. Baek, W.-H. Lee, J.-S. Kang, Y.-B. Jo, S. D. Lee, C.-K. Lee, J.-B. Choi, J.-H. Kim, K.-H. Park, W.-J. Cho, M.-G. Jang, and S.-J. Lee, IEEE Trans. Nanotechnol. 4, 242 (2005).

${ }^{7}$ P. Xiaodan and A. Broers, J. Appl. Phys. 71, 6189 (1992).

${ }^{8}$ J. R. Barnes, A. C. F. Hoole, M. P. Murrell, M. E. Welland, A. N. Broers, J. P. Bourgoin, H. Biebuyck, M. B. Johnson, and B. Michel, Appl. Phys. Lett. 67, 1538 (1995).

${ }^{9}$ D. V. Averin and K. K. Likharev, in Mesoscopic Phenomena in Solids, edited by B. Altshuler, P. Lee, and R. Webb (Elsevier, Amsterdam, 1991), pp. 173-271.

${ }^{10}$ G. L. Ingold and Y. V. Nazarov, in Single Charge Tunneling, edited by H. Grabet and M. H. Devoret (Plenum, New York, 1992), pp. 21-108.

${ }^{11}$ K. Matsumoto, M. Ishii, J.-I. Shirakashi, K. Segawa, Y. Oka, B. J. Vartanian, and J. S. Harris, Tech. Dig. - Int. Electron Devices Meet. 1995, 363.

${ }^{12}$ F.-C. Chiu, S.-K. Fan, K.-C. Tai, J. Y. Lee, and Y.-C. Chou, Appl. Phys. Lett. 87, 243506 (2005). 